\title{
Análisis comparativo del potencial de Biogás obtenido de la fracción Orgánica de residuos sólidos urbanos
}

\section{Comparative analysis of biogas potential obtained from the organic fraction of}

\section{solid urban waste.}

\section{Malacatus Cobos, Paúl, MSc.}

Docente Facultad de Ingeniería en Geología, Minas

Petróleos y Ambiental, Universidad Central del Ecuador pnmalacatus@uce.edu.ec

\section{Cristina Pantoja Vásquez}

Estudiante Facultad de Ingeniería en Geología, Minas, Petróleos y Ambiental, Universidad Central del Ecuador

\section{Jaime Marti}

Estudiante Facultad de Ingeniería en Geología, Minas,

Petróleos y Ambiental, Universidad Central del Ecuador

\section{Nathaly Cartuche Cojitambo}

Estudiante Facultad de Ingeniería en Geología, Minas,

Petróleos y Ambiental, Universidad Central del Ecuador

\section{Resumen}

La determinación de la técnica de digestión anaerobia más eficiente para el tratamiento de la FORS del Mercado de Carapungo del DMQ fue el objetivo del presente trabajo, mediante el estudio comparativo del potencial de biogás generado a partir de un biodigestor batch simple, un biodigestor batch con recirculación en una etapa y un biodigestor batch con recirculación en dos etapas. No se han realizado estudios comparativos entre diferentes técnicas de digestión anaerobia, evaluando el potencial de biogás obtenido de la fracción orgánica de residuos sólidos del Mercado de Carapungo. La hipótesis a comprobar fue si se genera mayor potencial de biogás a partir de la recirculación en dos etapas, en comparación con las otras técnicas de digestión anaerobia. El tratamiento de datos se realizó en Microsoft Excel para la comparación de las técnicas estudiadas, considerando el potencial de biogás, contenido de metano en el biogás y la complejidad del montaje de los biodigestores experimentales. La comparación del potencial de biogás entre las técnicas estudiadas batch no es posible dado que el gas obtenido posee un porcentaje de metano menor al 3\%, el cual no es representativo para ser considerado biogás. Se debe tratar la FORS del Mercado de Carapungo con sistemas de digestión anaerobia continuos, debido a que experiencias han demostrado que son más eficientes que los sistemas batch para este tipo de residuos.

Palabras clave: digestión anaerobia; fors; potencial de biogás; técnicas de digestión anaerobia batch

Abstract

Determine the most efficient anaerobic digestion technique for the treatment of the The determination of the most efficient anaerobic digestion technique for the treatment of the FORS of the Carapungo Market of DMQ was the objective of the present study, through the comparative study of the biogas potential generated from a simple batch biodigestor, a batch biodigestor with Recirculation in one stage and a batch biodigestor with recirculation in two stages. No comparative studies have been performed between different anaerobic digestion techniques, evaluating the biogas potential obtained from the organic fraction of solid waste in the Carapungo Market. The hypothesis to be verified was if it generates a greater potential of biogas from the recirculation in two stages, in comparison with the other techniques of anaerobic digestion. Data processing was performed in Microsoft Excel for the comparison of the studied techniques, considering the biogas potential, methane content in the biogas 
and the assembly complexity of the experimental biodigesters. The comparison of the biogas potential between the techniques studied batch is not possible since the obtained gas has a percentage of methane less than 3\%, which is not representative to be considered biogas. The FORS of the Carapungo Market must be treated with continuous anaerobic digestion systems, because experiences have shown that they are more efficient than batch systems for this type of waste.

Keywords: anaerobic digestion; biogas potential; ofsw; anaerobic digestion batch techniques

\section{Introducción}

En la parroquia de Carapungo existen proyectos de clasificación de los residuos sólidos para el aprovechamiento de los residuos sólidos inorgánicos, mientras que los residuos sólidos orgánicos son enviados al relleno sanitario. Es así que en el Mercado de Carapungo, la FORS no se somete a un ningún tipo de tratamiento (Administración Zonal Calderón, comunicado personal, 26 de octubre de 2014).

La presente investigación busco identificar la técnica que genere mayor potencial de biogás mediante diferentes técnicas de digestión anaerobia. De esta manera, este trabajo constituyo un material de consulta para futuras investigaciones y como base teórica para micro y macro proyectos de aprovechamiento energético.

\section{Material y métodos}

\section{Población y muestra}

El universo está constituido por la cantidad de residuos sólidos del Mercado de Carapungo, ubicado en la calle Río Cayambe y Neptay Godoy. La generación de residuos del mencionado mercado es de 2250 kg/semana, valor con el cual se estimó la generación diaria dividiéndolo para siete días. De esta manera se obtuvo un valor de $321 \mathrm{~kg} /$ día. Se toma una muestra representativa de $50 \mathrm{~kg}$, mediante la aplicación de la Norma Mexicana NMX-AA-15-1985, que establece el método de cuarteo para residuos sólidos municipales así como también la obtención de especímenes para el análisis de laboratorio.

\section{Instrumentos}

Para el montaje de los biodigestores se requirieron los siguientes elementos: 24 adaptadores de rosca a presión 3/4", 5 bidones de 15 gal, 2 bombas de acuario (16,2 I/min), 10 codos $\mathrm{HH} 90^{\circ} 3 / 4 ", 4$ gasómetros, 28 llaves, 5 neplos $6 \mathrm{~cm} 3 / 4$ ", 26 neplos $\mathrm{C} /$ tuerca 3/4", 18 neplos flex 3/4", 2 tapones hembra 3/4", 7 tees 3/4", 1 tees flex 3/4", 6 uniones R.R.3/4",
8 uniones universales 3/4", 6 cintas de teflón, 8m de manguera lisa, 1 sellador de caucho sintético, 1 timer, y 2 m de tubería 3/4".

Instrumentos para la preparación del inóculo.- Se utilizaron $50 \mathrm{~kg}$ de estiércol bovino del Centro Experimental Uyumbicho de la Facultad de Medicina Veterinaria y Zootecnia de la Universidad Central del Ecuador, 20 bolsas plásticas, 1 balanza, 2 palas, y 3 contenedores, 30 Botellas plásticas, 1 par de guantes de caucho, y 2 mascarillas protectoras.

Instrumentos para la caracterización de los residuos sólidos orgánicos del Mercado de Carapungo.- Se utilizaron $100 \mathrm{~kg}$ de residuos sólidos orgánicos del Mercado de Carapungo, 20 bolsas plásticas, 1 balanza, 1

par de guantes de carnaza, 2 escobas, 1 par de botas de hule, 2 mascarillas protectoras, y una superficie plana impermeable.

Instrumentos para las técnicas analíticas.-

- Determinación de humedad: termobalanza.- 1 espátula, 1 termobalanza OHAUS

- Determinación de humedad: Método de la cápsula de arena.- 1 Estufa, $100 \mathrm{~g}$ de arena, 12 cápsulas de aluminio, 12 palillos y 1 balanza.

Instrumentos para la determinación de sólidos totales (ST) y sólidos volátiles (SV).- 12 Crisoles, 1 Horno mufla, y 1 Balanza analítica.

Instrumentos para la determinación de $\mathrm{pH}$.- 5 vasos de precipitación, y $1 \mathrm{pH}$-metro Oakton, con una escala graduada en 0,005 unidades de $\mathrm{pH}$.

Instrumentos para el análisis de la composición del biogás.- 1 Cromatógrafo de gases, 4 fundas Tedlar, y 1 equipo de recolección de gas.

\section{Procedimiento}

Como primer paso se realizó el montaje de los bio- 
digestores experimentales para cada técnica de DA estudiada. Luego, la investigación se llevó a cabo en tres ensayos:

- Ensayo Uno: corresponde la validación de los biodigestores experimentales y preparación del inóculo.

- Ensayo Dos: corresponde al tratamiento de la FORS del Mercado de Carapungo. El análisis de biogás y $\mathrm{pH}$, realizado al final de este ensayo, constató la acidificación de los biodigestores, por lo que se desarrolló el Ensayo Tres.

- Ensayo Tres: corresponde a la validación de resultados.

A continuación se detalla la realización de cada ensayo:

Montaje de los biodigestores para las tres técnicas de digestión anaerobia.

Se montaron cuatro biodigestores, que constituyen cuatro sistemas, diseñados para llevar a cabo los ensayos en modo batch. Estos reactores tienen un diámetro de 0,35 $\mathrm{m}$, altura de 0,63 $\mathrm{m}$ y un volumen total de $60 \mathrm{~L}$.

- Biodigestor 1: En este biodigestor se instalaron válvulas para toma de muestras ubicadas en distintos niveles del bidón, además se instaló un medidor de gas en la parte superior.

- Biodigestor 2: En este biodigestor, al igual que el anterior, se instalaron válvulas para toma de muestras ubicadas en distintos niveles del bidón y un medidor de gas en la parte superior.

- Biodigestor 3: Además de las válvulas para toma de muestras ubicadas en distintos niveles del bidón, y el medidor de gas instalado en la parte superior, este biodigestor cuenta con una bomba encerrada dentro de una malla, la cual está ubicada en la parte inferior del biodigestor. La finalidad de esta bomba es recircular el lixiviado generado, a través de una manguera que conecta el fondo del biodigestor con una ducha instalada en la parte superior, de esta manera se obtiene la recirculación en una etapa.

- Biodigestor 4: En este biodigestor, ambas etapas están conectadas entre sí por la parte superior a través de una manguera y por la parte inferior a través de 2 mangueras, para favorecer la recirculación en dos etapas. La primera etapa está destinada a la digestión del sustrato sólido. Una vez que genera lixiviado es llevado por las mangueras inferiores hacia la segunda etapa, para lo cual se llenó de botellas de plástico para favorecer la formación de biopelícula. Ésta última está destinada al tratamiento únicamente del líquido. Cuando la segunda etapa se llena, el líquido se recircula hacia la primera etapa, para continuar la recirculación.

Ensayo Uno: Validación de los dispositivos experimentales y preparación del inóculo

En este ensayo se aseguró la hermeticidad de los dispositivos experimentales mediante varias alternativas de sellado, y a la vez se preparó el inóculo para el Ensayo Dos.

Los biodigestores experimentales debieron cumplir con condiciones anaerobias para permitir la generación del biogás. Se logró que los biodigestores sean herméticos después de probar las siguientes opciones, y con cada una se verificó la hermeticidad a través de la medición de biogás en cada dispositivo.

a) Teflón en el borde de la tapa del bidón.

b) Silicona en el borde de la tapa del bidón.

c) Plástico en el borde de la tapa del bidón.

d) Volteo de biodigestores.

Luego de 77 días de monitoreo, se estimó pertinente un cambio en la posición de los biodigestores para favorecer su hermeticidad. Es así que se voltearon los biodigestores para que el peso del contenido de los mismos recaiga sobre la tapa previamente cerrada. De esta manera, se creó un sello de agua que impide una posible fuga de biogás.

Pese a que el estiércol de porcino tiene mayor producción de biogás que el de bovino (Qiao et al., 2011), en este proyecto de investigación se ha utilizado únicamente como inóculo estiércol de bovino, dada la accesibilidad y facilidad para su recolección y transporte por parte del Centro Experimental Uyumbicho hasta el laboratorio de biomasa del INER ubicado en Carapungo.

Una vez que se abrieron las bolsas plásticas, se homogenizó el contenido de las mismas. Luego se llenó cada biodigestor (B1, B2, B3, B4) con 10 kg de estiércol. Posteriormente se diluyó el estiércol con agua potable con una relación 1:2,5 para mejorar el flujo del sustrato cuando es recirculado con la bomba. Después, se clasificaron 50 botellas plásticas por tamaño con el fin de calcular la superficie total 
de contacto (3,39 m2), éstas fueron cortadas en tres partes y colocadas en el interior del digestor B4 para que los microorganismos se fijen a dicha superficie y formen una biopelícula. Finalmente se cerraron los biodigestores y se monitoreó hasta que se detectó la producción de biogás mediante los gasómetros.

Ensayo Dos: Tratamiento de la FORS del Mercado de Carapungo

Antes de llevar a cabo el tratamiento de los residuos sólidos orgánicos del Mercado de Carapungo, fue necesario caracterizarlos para conocer su composición.

\section{- Toma de muestras}

El día jueves, 22 de enero de 2015, se recolectaron $100 \mathrm{~kg}$ de residuos en el Mercado de Carapungo, los mismos que fueron transportados al laboratorio de biomasa del INER. Se separaron los residuos sólidos orgánicos de los inorgánicos, y éstos a su vez en residuos orgánicos de frutas y verduras y papel/cartón debido a que estos últimos no se los ha tomado en cuenta para la alimentación de los biodigestores por su alto contenido de lignocelulosa. Es así que la cantidad de muestra disponible para realizar el cuarteo fue de $72,72 \mathrm{~kg}$. Luego se obtuvo una muestra para la selección y cuantificación de subproductos, y otra para los análisis de laboratorio, siguiendo el procedimiento descrito en la Norma Mexicana NMX-AA-15-1985. Éste método consiste en depositar los residuos recolectados en un área plana e impermeable para formar una pila, mezclar aleatoriamente los residuos e igualar las características en todas las partes de la pila. Posteriormente, la pila de residuos se divide en cuatro partes iguales, de las cuales dos partes opuestas se eliminan y el resto se vuelve a homogeneizar repitiendo el proceso hasta obtener una muestra representativa del residuo. Aunque la Norma Mexicana menciona que el proceso de cuarteo debe ser repetido hasta dejar un mínimo de $50 \mathrm{~kg}$ de residuos sólidos, se ha obtenido una muestra de 19,246 kg debido a que la cantidad de residuos disponibles para dicho fin fue de $72,72 \mathrm{~kg}$, a los cuales se realizó el cuarteo por dos veces.

Previamente de las partes eliminadas del primer cuarteo, se tomaron $10 \mathrm{~kg}$ aproximadamente de residuos sólidos para los análisis de laboratorio y con el resto se determinó la densidad de los residuos sólidos "in situ".

- Cuantificación de subproductos
Una vez obtenida la muestra de los residuos orgánicos del Mercado de Carapungo se separaron en subproductos para conocer la composición de dichos residuos, los cuales fueron colocados dentro de los biodigestores. De acuerdo a la Norma Mexicana NMX-AA-22-1985, los productos ya clasificados se pesan por separado en la balanza y se anota el resultado en la hoja de registro con la finalidad de calcular el porcentaje en peso de cada uno de los subproductos, que se calcula con la siguiente expresión:

$$
P S_{i}=\frac{G_{i}}{G} * 100 \quad \text { Ec. (1) }
$$

$$
\begin{aligned}
& \text { En donde: } \\
& P S_{i}=\text { porcentaje del subproducto considerado } \\
& G_{i}=\text { peso del subproducto considerado }(\mathrm{kg}) \text {; }
\end{aligned}
$$

descontando el peso de la bolsa empleada

$\mathrm{G}=$ peso total de la muestra

La misma norma hace referencia que al sumar los diferentes porcentajes, debe ser como mínimo el 98\% del peso total de la muestra $(G)$. En caso contrario, se debe repetir la determinación.

\section{- Densidad in situ}

Según la Norma Mexicana NMX-AA-19-1985, de las partes eliminadas del primer cuarteo se toma una cantidad de residuos sólidos para medir la densidad "in situ", que en la presente investigación fue de $12 \mathrm{~kg}$.

Con un recipiente de aproximadamente $10 \mathrm{~L}$ y de $561 \mathrm{~g}$ de peso, se adicionaron los residuos sólidos homogenizados hasta llenar el recipiente y se golpeó el recipiente contra el suelo tres veces dejándolo caer desde una altura de $10 \mathrm{~cm}$. Después se agregaron los residuos sólidos hasta volver a llenar el recipiente, sin ejercer ninguna presión cuando se los coloque en el recipiente, con el fin de no alterar la densidad que se pretende determinar. El peso neto de los residuos es el valor del peso del recipiente restado el de la tara, y la densidad del residuo sólido orgánico se calcula mediante la siguiente expresión:

$$
\rho=\frac{p}{V} * 100 \quad \text { Ec. (2) }
$$

$\rho$ : Densidad del residuo $(\mathrm{kg} / \mathrm{m} 3)$

$P$ : Peso de los residuos $(\mathrm{kg})$

$\mathrm{V}$ : Volumen del recipiente (m3) 
Es así que, se obtuvo $30 \mathrm{~kg}$ de residuos de las partes eliminadas de ambos cuarteos, los mismos que se cargaron a los biodigestores para su tratamiento.

- Carga del sustrato e inóculo en los biodigestores experimentales

El biodigestor B1 fue cargado con inóculo y agua en una relación de 1:2 para que sirva de testigo en la comparación con los demás biodigestores estudiados. Se cargaron 8,3 L de inóculo con 16,6 $\mathrm{L}$ de agua. Por otro lado, los biodigestores B2, B3 y B4 fueron cargados con residuos sólidos orgánicos del Mercado de Carapungo e inóculo diluido en agua con una relación 1:1. Se empleó esta relación FORS-inóculo para tener un valor intermedio de las proporciones de la bibliografía estudiada, como es el caso de Martí-Herrero et al. (2014) que llega a utilizar 100\% de FORS del Mercado de Tiquipaya en Bolivia y la relación de Castelo (2012) que emplea un 5\% de la mezcla de residuos de frutas y vegetales del Mercado Central de Tumbaco. Se cargaron $10 \mathrm{~kg}$ de residuos sólidos orgánicos, 8,3 L de inóculo y 16,6 L de agua.

\section{Ensayo Tres: Validación de resultados}

Nuevamente, se recolectó estiércol de bovino del Centro Experimental Uyumbicho, con las mismas características del estiércol utilizado en el Ensayo Uno. La carga del estiércol y agua, en los biodigestores se realizó mediante un procedimiento similar al descrito en el Ensayo Uno, disminuyendo la relación estiércol-agua a 1:1. Se cargaron $20 \mathrm{~kg}$ de estiércol con $21 \mathrm{~L}$ de agua. En este ensayo no se recircularon los biodigestores B3 y B4 para observar el comportamiento de todos los sistemas en condiciones iguales, es decir como sistemas batch.

Para determinar las características del sustrato, en el laboratorio se analizaron parámetros como $\mathrm{pH}$, temperatura, humedad, sólidos volátiles, concentración de metano, carbono orgánico, nitrógeno total, fósforo total y potasio.

\section{- Humedad: método de la termobalanza}

Según lo establecido en la norma mexicana NMX-F-428- 1982, para la determinación rápida de la humedad en alimentos es posible el uso de una termobalanza que analizará la muestra de los residuos sólidos orgánicos y determinará su humedad, requerida para la determinación de sólidos volátiles. El procedimiento es el establecido a continuación:
Primero se pesa una cantidad de muestra según lo requerido por el equipo, en este caso entre 0,9 y 1,0 gramos, se cierra el analizador de humedad y este determina el peso exacto de la muestra. A continuación, la muestra se calienta por medio de la unidad desecadora y la humedad se evapora.

Durante la operación de desecación, el equipo determina continuamente el peso de la muestra, y una vez terminada la desecación, el resultado se muestra como porcentaje de contenido de humedad.

- Humedad: método de la cápsula de arena

La humedad del estiércol fue determinado a través del método de la cápsula de arena, el cual permite el análisis de muestras líquidas o con gran cantidad de humedad. Según lo establecido por Nielsen (2010), el procedimiento inicia calcinando la arena de mar para luego colocar entre 10 y 30 gramos de la misma en las cápsulas de aluminio limpias además de un palillo. Después estas cápsulas se llevan a la estufa a $103^{\circ} \mathrm{C}$ durante dos horas. Una vez transcurrido este tiempo se enfrían en un desecador hasta temperatura ambiente y se pesan en una balanza las cápsulas que contienen la arena y el palillo. Se coloca en la cápsula con la arena y el palillo, entre 5 y $10 \mathrm{~g}$ demuestra y se la mezcla con la arena de forma que quede bien disgregada y no se forme una costra superficial al calentarse. Se introduce las cápsulas a la estufa a $103^{\circ} \mathrm{C}$ durante 3 a 6 horas, después de saca de la estufa y se deja enfriar hasta temperatura ambiente cuando las cápsulas son pesadas en la balanza. Este último paso se repite hasta que dos pesadas consecutivas sean constantes.

El contenido en agua de la muestra se calcula por diferencia de peso y se expresa en porcentaje de humedad (g de H2O/100 g de muestra) (Nielsen, 2010): 


\section{- Sólidos volátiles}

Los sólidos volátiles (SV), fueron determinados a través del método establecido en la Norma Británica BS EN 15148:2009, la cual sugiere el procedimiento descrito a continuación: 5 crisoles vacíos se colocaron en el horno a $900^{\circ} \mathrm{C}$ por $7 \mathrm{mi}-$ nutos para ser tarados, luego se pesó cada uno (m1). Después se pesó 1 gramo de una muestra previamente homogeneizada (m2). A continuación, se colocó estas muestras nuevamente en el horno durante 7 minutos. Finalmente se pesa el crisol con la muestra (m3). Los valores m1, m2, m3 y $\mathrm{H}$ se reemplazan en la siguiente fórmula para el cálculo de los sólidos volátiles.

$$
S V=\frac{100 m_{2}-m_{3}}{m_{2}-m_{1}}-H^{*} \frac{100}{100-H} \quad \text { Ec. (4) }
$$

Donde:

$m_{1}$ : peso del crisol tarado

$m_{2}$ : peso del crisol +1 gramo de muestra del residuo

sollido

$m_{3}$ : peso final

$\mathrm{H}$ : humedad

\section{- Determinación de pH}

La Norma Internacional ISO 1842:1991, referente a la determinación de $\mathrm{pH}$ en vegetales y frutas, señala que antes de sumergir el electrodo del equipo se prepara la muestra de ensayo. Para productos líquidos y productos fácilmente filtrables, se mezcla la muestra cuidadosamente hasta que esté homogénea. Para productos espesos o semi espesos y productos de los cuales es difícil separar el líquido, se mezcla una parte de la muestra y se la muele, si es necesario, en un mezclador o mortero. Si el producto obtenido todavía es muy espeso, se añade una masa equivalente de agua destilada y si es necesario, se mezcla bien con un mezclador o mortero. Luego, con el pH-metro previamente calibrado, se determina el pH por lectura directa, introduciendo el electrodo del $\mathrm{pH}$-metro en el vaso de precipitación con la muestra preparada. La determinación debe efectuarse por duplicado y como resultado final se reporta la media de los resultados obtenidos.

\section{- Monitoreo de temperatura}

El monitoreo de temperatura se realizó a partir del Ensayo
Dos, mediante la colocación de un sensor de temperatura en el interior del biodigestor B2. Este sensor fue programado para una lectura de temperatura cada hora. Al final de cada ensayo se obtuvo el registro de temperatura, se calculó la media de temperatura diaria. Después, se construyó una gráfica Temperatura-Tiempo de Retención, con lo cual se determinó en qué rango de temperatura se llevó a cabo la digestión anaerobia.

\section{- Tiempo de retención}

El tiempo de retención (TR) en cada uno de los ensayos fue contado a partir del día cero, en el cual se carga el sustrato, hasta el último día en el cual dicho sustrato fue descargado.

\section{- Composición de biogás}

La composición del biogás se analizó en un cromatógrafo de gases, siguiendo al protocolo del laboratorio de biomasa del INER. Se abrió la llave de paso de gas en el biodigestor para dejar correr una mínima parte de gas con el objeto de obtener una muestra homogénea en las fundas Tedlar. Se conectó un tapón acoplado a una pequeña manguera que entra en el equipo de recolección de gas y se lo conecta a la funda Tedlar. Se cierra el equipo de recolección de gas y se prende para recolectar la muestra. El procedimiento se repite para cada biodigestor. En el cromatógrafo, se programa el análisis de gas con tres repeticiones, se acopla la funda de muestra al equipo y se deja correr el programa. Después de las tres repeticiones, se obtienen los datos de la composición de biogás en porcentaje de metano.

\section{Determinación del potencial generado a partir de las ex- perimentadas}

La medición de biogás tomada de los gasómetros instalados en cada biodigestor fue registrada diariamente. En una hoja de Excel se utilizaron los datos para crear las gráficas de producción de biogás de cada biodigestor y facilitar la comparación entre ellas. Este procedimiento fue realizado hasta obtener una producción de biogás aproximadamente constante, es decir, una asíntota en la gráfica de producción acumulada de biogás.

La producción acumulada de biogás para cada biodigestor es normalizada a una atmósfera de presión y 273,15 K de temperatura para estan- 
darizar los datos y que estos sean comparables con otras investigaciones. El último dato normalizado de producción acumulada de biogás de cada biodigestor fue utilizado en la ecuación 1 descrita en el apartado 2.4.1 correspondiente a la producción específica de biogás (PEB).

\section{Metodología para el análisis de datos}

Los parámetros analizados fueron tratados a través del uso de medios informáticos como hojas electrónicas en Microsoft Excel, para de esta manera facilitar su orden cronológico, así como el cálculo, procesamiento e interpretación gráfica de los mismos.

Además, para la comparación de las técnicas estudiadas se deben considerar variables como el potencial de biogás, la concentración de metano en el biogás y la complejidad del montaje de los biodigestores experimentales, con la finalidad de identificar la mejor técnica para el tratamiento anaerobio de los residuos sólidos orgánicos en cuestión.

\section{Resultados}

\section{Características de los residuos sólidos orgánicos genera- dos en el mercado de carapungo}

de los $100 \mathrm{~kg}$ de los residuos del Mercado de Carapungo, luego de la separación de los residuos inorgánicos y de los residuos de papel y cartón, se obtuvo $72,72 \mathrm{~kg}(72,72 \%)$ de residuos de frutas y verduras para la posterior aplicación del método de cuarteo. Adicionalmente, se obtuvo 3,67\% de papel y cartón, y 23,61\% de inorgánicos. El porcentaje de residuos sólidos orgánicos de frutas y verduras para el Mercado de Carapungo (73\%) es relativamente menor al obtenido por Castillo (2012) en la caracterización de residuos sólidos de mercado a nivel de Quito (83,6\%).

Una vez realizada esta actividad, se determinaron las características de la FORS del Mercado de Carapungo.

\section{- Cuanti icación de subproductos}

De los 19, $246 \mathrm{~kg}$ de residuos obtenidos de la aplicación del cuarteo, se seleccionaron, se separaron los subproductos, se pesaron y se obtuvo el porcentaje en peso de cada uno, aplicando la ecuación 1. El error obtenido en la cuantificación de subproductos es inferior al $2 \%$, valor máximo establecido por la norma mexicana utilizada para dicho fin.

El residuo presentó un alto porcentaje de frutas y verduras ya que supone alrededor del $90 \%$ del peso total de la muestra. Por último, se puede destacar que el residuo del Mercado de Carapungo tiene gran cantidad de cáscara de plátano $(27,76 \%)$, plantas medicinales (17,43\%), papa/ yuca $(10,24 \%)$, cítricos/ cáscara de piña (15,58\%). La presencia de cítricos en la FORS puede inhibir la digestión anaerobia, ya que los aceites esenciales de su corteza son citotóxicos (Ruiz \& Flotats, 2014).

\section{- Densidad in situ}

Para la determinación de la densidad "in situ" se tomó una fracción del residuo eliminado en el primer cuarteo. Conociendo el volumen del recipiente (10 L) y el peso del recipiente lleno de residuos (restado el peso del recipiente), se obtuvo la densidad del residuo con reemplazando dichos valores en la ecuación 2.

$$
\rho=\frac{3612}{10 L}=316,2 g / L
$$

$\left(\mathrm{kg} / \mathrm{m}^{3}\right)$

Este parámetro está dentro del rango esperado, ya que de acuerdo con Kurup (2003), la densidad media de los residuos sólidos orgánicos corresponde a un valor entre 200 a $500 \mathrm{~kg} /$. Un valor similar fue obtenido por Álvaro \& Olives (2013), que determina una densidad promedio de $320 \mathrm{~kg} / \mathrm{m} 3$ para los mercados de la Zona Sur del DMQ, mientras que CastiIlo (2012) presenta un valor de 233,66 kg/m3 para el Mercado del Camal en Quito.

\section{- Características químicas de la FORS}

El valor de sólidos volátiles de la FORS $76,01 \%$ es un indicativo de una alta concentración de materia orgánica susceptible al tratamiento anaerobio. Este valor coincide con los valores recopilados por Lohri (2009) para residuos de mercado que está dentro del rango $75-90 \%$. Sin embargo, según lo establecido por Díaz de Basurto (2013), este valor es menor comparado con los valores de sólidos volátiles obtenidos en los mercados de La Paz y El Alto, 95,77\% y $94,51 \%$, respectivamente.

Se obtuvo un $\mathrm{pH}$ de 7, que demuestra una condición favorable para el funcionamiento de los biodigestores, y es comparable con los valores de $\mathrm{pH}$ obtenidos para la ciudad de La Paz y El Alto, mencionados en Díaz de Basurto (2013), 7,0 y 7,2 respectivamente.

Por su parte, la cantidad de nutrientes es indispensable para el crecimiento de los microorganismos encargados de la DA. Para la FORS se obtuvo un valor de nitrógeno total de $0.2831 \%$, el cual difiere en gran medida con los valores reportados por Díaz 
de Basurto (2013) para las ciudades de La Paz y E Alto, 3,09\% y $3.47 \%$, respectivamente. Mientras, el valor de carbono orgánico de 9,95\%, que comparado con el valor reportado por Sitorus (2013) 22,27\%, resulta bajo. De todas formas, la relación de estos elementos 35.1/1 se encuentra dentro de los valores recomendables (15/1 - 45/1), según lo establecido por Elías et al. (2012).

De igual manera, el valor de fósforo total $0.4022 \%$, es muy similar al reportado para la ciudad de El Alto, con 0,32\% (Díaz de Basurto, 2013).

\section{Parámetros ambientales y de control}

\section{- Determinación de pH}

El pH fue monitoreado en cada uno de los ensayos. En el Ensayo Uno se tomaron dos valores de $\mathrm{pH}$, el inicial (estiércol) y el final (inóculo) para uno de los reactores (B1). Para el Ensayo Dos se tomaron medidas de $\mathrm{pH}$ del inóculo, FORS y del biol, para cada uno de los biodigestores. Mientras que para el Ensayo Tres la medición de $\mathrm{pH}$ se realizó semanalmente.

Ensayo Uno: En este ensayo se tomó el pH en dos ocasionales en un sólo biodigestor (B1) ya que todos contenían el mismo material. La primera, del estiércol, con un valor de 8,5 y la segunda del inóculo después de 83 días, reportando un valor de 7,11. Para el caso de los cuatro Biodigestores, el pH descendió de 8,5 a 7,11, valores comparables con lo obtenido por Hidalgo \& Lara (2011), quienes reportan un $\mathrm{pH}$ de 9 antes del tratamiento y un valor de 7,1 después del mismo.

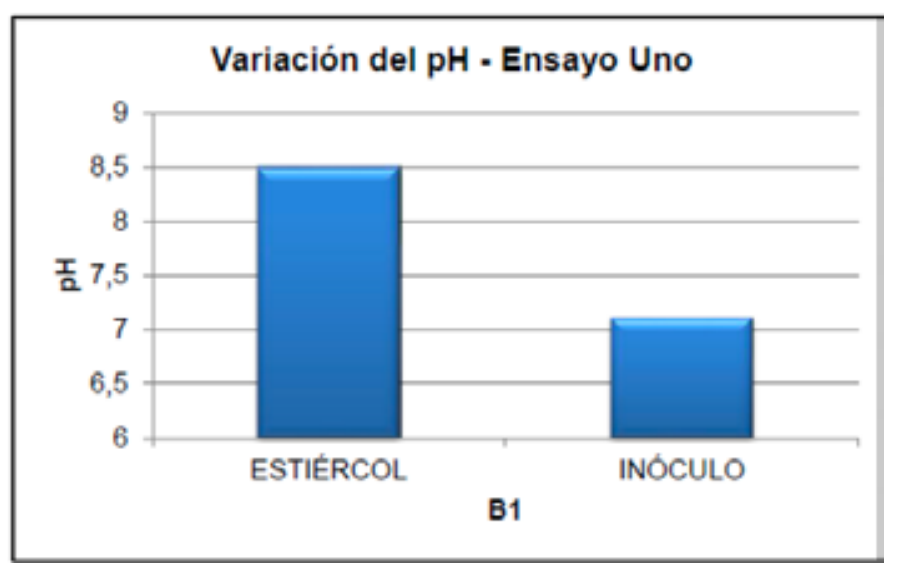

Figura 1 Comparación del pH inicial y final biodigestor 1- Ensayo Uno / Elaborado por: Malacatus Paúl \& all.

Ensayo Dos: El pH inicial corresponde al valor de $\mathrm{pH}$ de la muestra de FORS del Mercado de Carapungo y al $\mathrm{pH}$ del inóculo preparado en el Ensayo Uno, mientras que el $\mathrm{pH}$ final es el valor obtenido luego de la DA correspondiente a la muestra de biol para cada biodigestor, como se indica en la siguiente tabla:

\section{Tabla 1 Variación de pH-Ensayo Uno}

\begin{tabular}{|c|c|c|c|c|c|c|}
\hline \multicolumn{2}{|c|}{ Biodigestores } & \multirow{2}{*}{ B1 } & \multirow{2}{*}{ B2 } & \multirow{2}{*}{ B3 } & \multicolumn{2}{c|}{ B4 } \\
\cline { 5 - 8 } & & & & etapa 1 & etapa 2 \\
\hline \multirow{2}{*}{ PH inicial } & FORS & & 7 & 7 & 7 & 7 \\
\cline { 2 - 8 } & INOCULO & 7,11 & 7,11 & 7,11 & 7,11 & 7,11 \\
\hline PH final & BIOL & 6,63 & 4,75 & 4,75 & 4,82 & 4,82 \\
\hline
\end{tabular}

Elaborado por: Malacatus Paúl \& all.

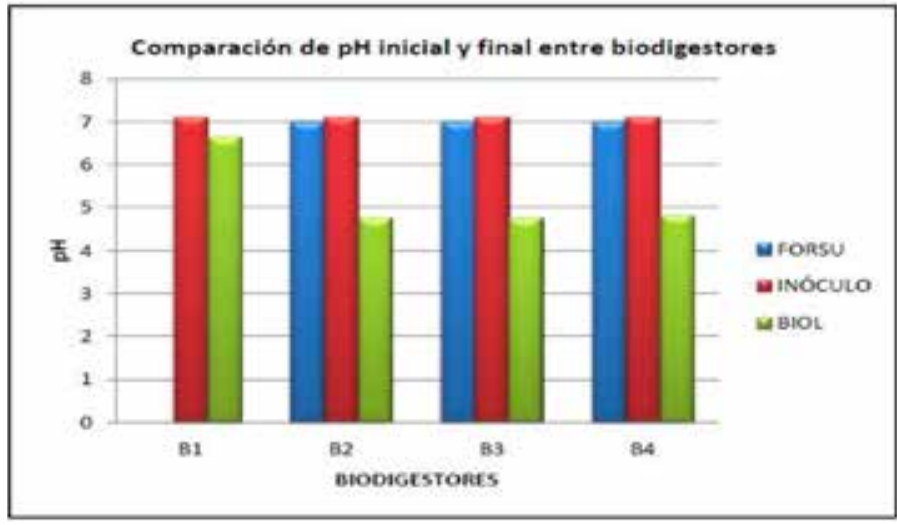

Figura 2 Comparación del pH inicial y final entre biodigestores - Ensayo Dos / Elaborado por: Malacatus Paúl \& all.

Respecto al biodigestor B1, el cual contenía únicamente inóculo de estiércol de bovino, el pH descendió de 7,11 a 6,63, el cual se acerca a la neutralidad. Varnero (2001 citado en FAO, 2011) reporta un valor de $\mathrm{pH}$ promedio de 7,9 para biol proveniente de digestores batch, en tanto de Hidalgo \& Lara (2011) determinan un $\mathrm{pH}$ entre 7,1 y 7,33 para la misma clase de reactor.

En cuanto a los biodigestores B2, B3 y B4, alimentados con FORS, el pH descendió a 4,75; 4,75 y 4,82 respectivamente. La disminución de $\mathrm{pH}$ conlleva a una digestión anaerobia ácida, provocada por una alta producción de ácidos grasos volátiles (Rittmann \& McCarty, 2001 citado en Díaz de Basurto, 2013). Adicionalmente, la FORS tuvo un porcentaje considerable de cítricos y cáscara de piña $(15,58 \%)$. Bogotá et al. (2008) también reporta un descenso de $\mathrm{pH}$ a 4,7 al final de la DA de la FORS del Mercado "Plaza Keneddy" en Bogotá.

Ensayo Tres: Se monitoreó el pH durante 37 días, semanalmente como se muestra en el Anexo J. El $\mathrm{pH}$ inicial corresponde al valor de $\mathrm{pH}$ de la muestra de estiércol fresco, mientras que el $\mathrm{pH}$ final es el valor obtenido luego de la DA correspondiente a la muestra de inóculo para cada biodigestor, como se indica en la siguiente tabla:

Tabla 2 Variación de pH-Ensayo Tres

\begin{tabular}{|c|c|c|c|c|c|c|}
\hline \multirow{2}{*}{ BIODIGESTORES } & \multirow{2}{*}{ B1 } & \multirow{2}{*}{ B2 } & \multirow{2}{*}{ B3 } & \multicolumn{2}{|c|}{ B4 } \\
\cline { 5 - 7 } & & & & etapa 1 & etapa 2 \\
\hline INICIAL & ESTIÉRCOL & 8,5 & 8,5 & 8,5 & 8,5 & 8,5 \\
\hline FINAL & INÓCULLO & 7,0 & 6,6 & 6,6 & 6,8 & 6,2 \\
\hline
\end{tabular}


Elaborado por: Malacatus Paúl \& all. Fuente: Malacatus Paúl \& all.

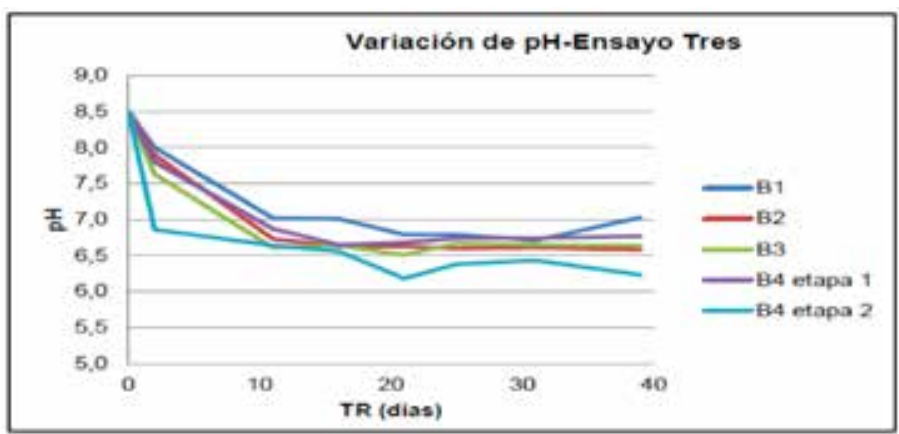

El pH del sustrato de los cuatro biodigestores se mantuvo cerca de la neutralidad, lo que no ocurrió con los biodigestores cargados con FORS del Ensayo Dos. Por lo tanto, la composición de la FORS inhibió el proceso de DA.

\section{- Monitoreo de temperatura}

Ensayo Dos: La temperatura se monitoreó en el Ensayo Dos durante 66 días, y como se puede verificar en la figura 17 , la temperatura promedio fue $18,81^{\circ} \mathrm{C}$, con una temperatura máxima de $20,15^{\circ} \mathrm{C}$ y una mínima de $16,29^{\circ} \mathrm{C}$. Por lo tanto se puede afirmar que el proceso de DA se mantuvo en un rango psicrofílico.

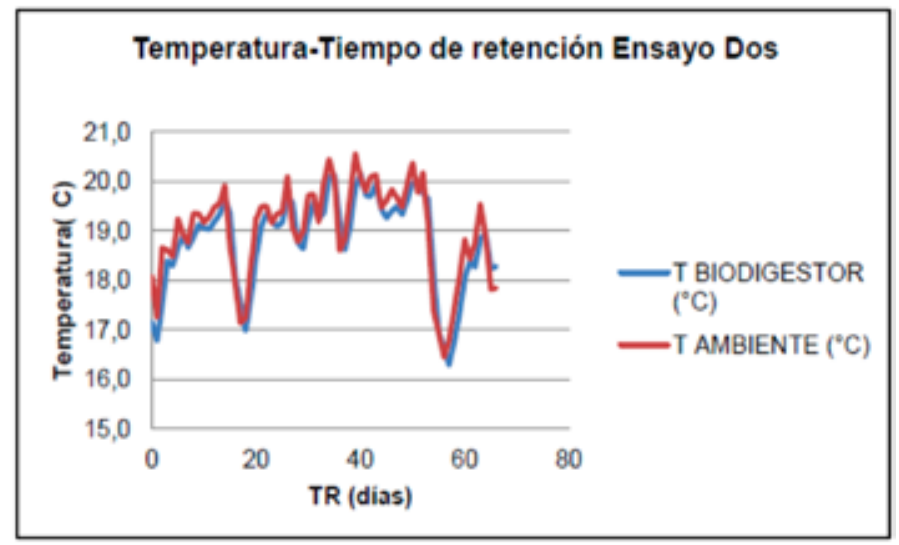

Figura 4 Gráfica Temperatura-Tiempo de retención Ensayo Dos Elaborado por: Malacatus Paúl \& all.

El experimento para el Ensayo Dos se llevó a cabo en 66 días, y de acuerdo con Lagrange (1979 citado en FAO, 2011), en rangos psicrofílicos, el TR requerido para completar la DA puede llegar a sobrepasar los 100 días. Es así que, Bogotá et al. (2008), con un TR de 120 días, alcanzó una temperatura similar de $18^{\circ} \mathrm{C}$ en el proceso de DA.

Por otra parte, para un ótpimo funcionamiento del digestor, las variaciones de temperatura dentro del reactor no deben exceder los $0.6-1.2^{\circ} \mathrm{C} /$ día., dado que variaciones bruscas de temperatura puede inducir la desestabilización del proceso (FAO, 2011).
Como se puede verificar en el Anexo F, en el Ensayo Dos hubieron valores de variación de temperatura $\left(1,5^{\circ} \mathrm{C}\right)$ que sobrepasaron ese rango.

Ensayo Tres: En este ensayo, la temperatura se monitoreó durante 42 días, y como se puede verificar en la figura 18 , la temperatura promedio fue 18,40 ${ }^{\circ} \mathrm{C}$, con una temperatura máxima de $19,60^{\circ} \mathrm{C}$ y una mínima de $16,65^{\circ} \mathrm{C}$. De esta manera se afirma que el proceso de DA también se mantuvo en un rango psicrofílico.

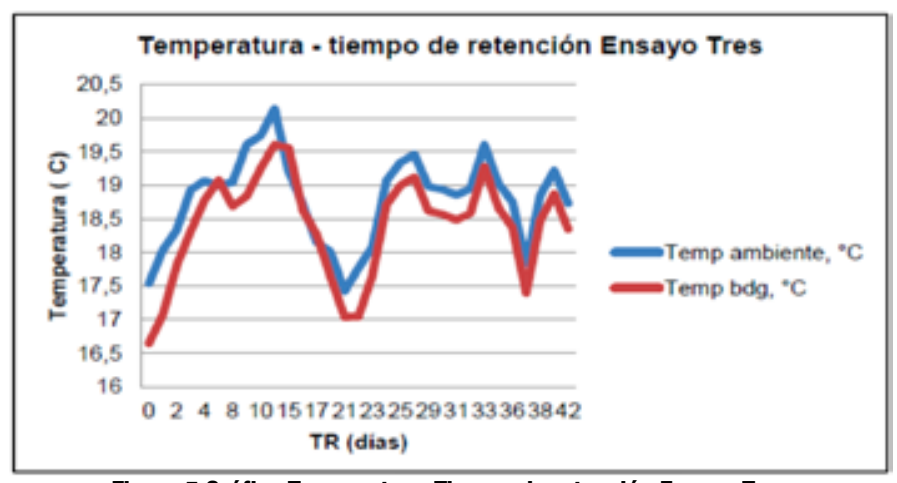

Figura 5 Gráfica Temperatura-Tiempo de retención Ensayo Tres

\section{ANÁLISIS COMPARATIVO DE DATOS}

- Potencial de biogás a partir de las técnicas de digestión anaerobia utilizadas

Ensayo Uno: El Ensayo Uno correspondiente a la validación de los dispositivos experimentales, se llevó a cabo en 83 días, ya que desde un inicio se presentaron problemas en la producción de biogás en los biodigestores, como se puede constatar en la siguiente figura.

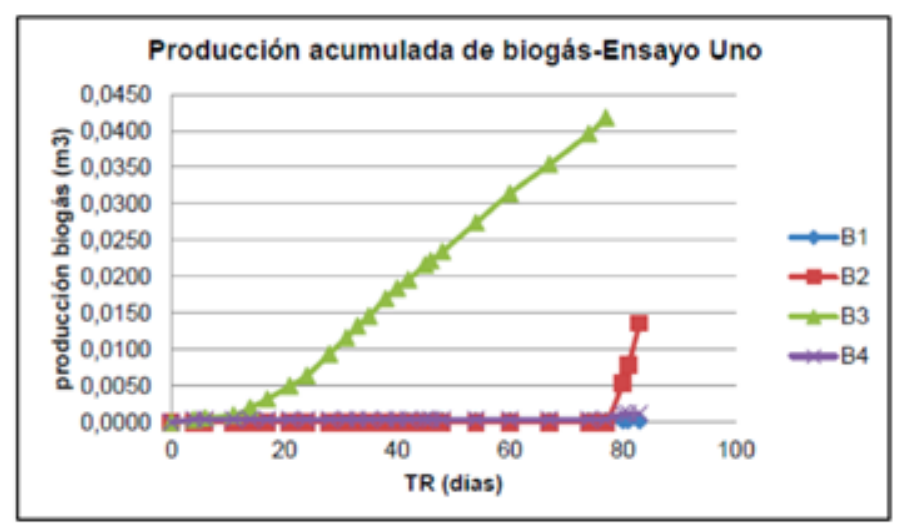

Figura 6 Producción acumulada de biogás-Ensayo Uno

Se logró que los biodigestores sean herméticos en el día 77 con el volteo de los mismos, ya que el biodigestor B2 registró una variación en la producción de biogás. Por otro lado, la tendencia lineal del biodigestor B3 muestra que en el momento de la recirculación, se desplazaba aire, cuyo volumen fue registrado por el gasómetro y por lo tanto no se trataba de biogás. 
La composición de biogás del Ensayo Uno no fue analizado porque la finalidad del mismo fue obtener el inóculo para el Ensayo Dos.

Ensayo Dos: La producción acumulada de biogás obtenido del tratamiento de la FORS del Mercado de Carapungo, fue monitoreado durante 66 días. A continuación se presenta una gráfica de la producción obtenida:

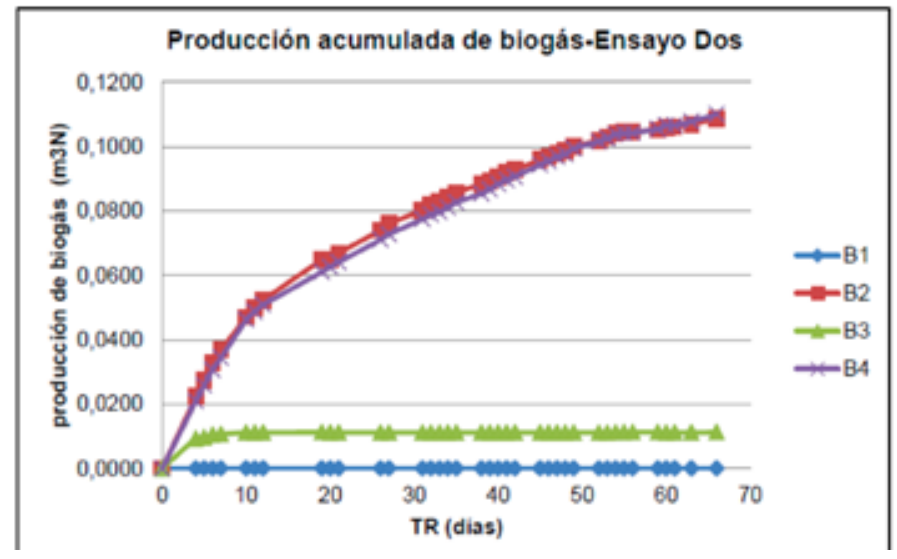

Figura 7 Producción acumulada de biogás-Ensayo Dos

A pesar de que aparentemente se validó la hermeticidad de los dispositivos experimentales, el biodigestor B1 no produjo biogás y el biodigestor B3 sólo produjo hasta el día 10. Por su parte, los biodigestores B2 y B4 tuvieron un comportamiento similar hasta el día 10. Del día 10 al día 50 se observa que el biodigestor B2 ligeramente produce mayor volumen de biogás en comparación con B4, pero a partir del día 50, B2 y B4 tienen aproximadamente la misma producción.

Para el cálculo del potencial de biogás se utilizó la siguiente ecuación:

$$
\operatorname{PEB}(B 2)=\frac{0,1088 \mathrm{~m}^{3} \mathrm{~N}}{7,601 \mathrm{kgSV}}=0,0143 \frac{\mathrm{m}^{3} \mathrm{Nbiogás}}{\mathrm{KgSV}}
$$

Se obtuvo 0,0143 $\mathrm{m}^{3} \mathrm{~N} / \mathrm{kgSV}$ en el Biodigestor 2 $0,0145 \mathrm{~m}^{3} \mathrm{~N} / \mathrm{kgSV}$.

En la siguiente gráfica, se representa la producción de biogás específica con respecto al TR de las diferentes técnicas estudiadas para este ensayo.

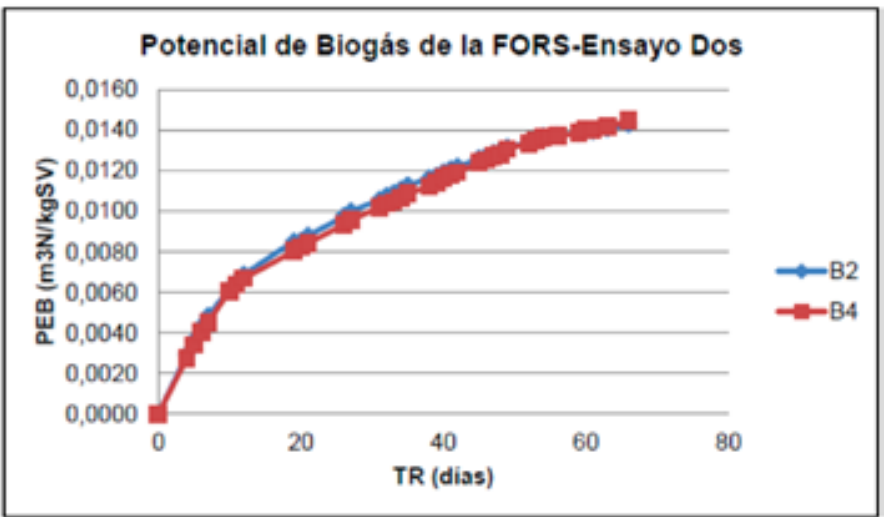

Figura 8 Potencial de biogás de la FORS-Ensayo Dos Elaborado por: Malacatus
Como se puede observar, los valores de PEB en los biodigestores B2 y B4 son muy similares, lo cual indica que el modo de funcionamiento establecido para el B2 (batch) y el B4 (recirculación en dos etapas) no supone una diferencia significativa en producción específica de biogás.

El cese en la producción de B3 se puede justificar ya sea por daños en el sistema de recirculación o por problemas de falta de hermeticidad, lo cual ha sido validado con el Ensayo Tres.

Por otra parte, se determinó el porcentaje de metano de las muestras del biogás de cada digestor, cuyos resultados se resumen en la siguiente tabla:

\section{Tabla 3 Concentración de metano-Ensayo Dos}

\begin{tabular}{|c|c|c|c|c|c|c|}
\hline \multirow{2}{*}{ COMPONENTE } & \multirow{2}{*}{ UNIDAD } & \multicolumn{4}{|c|}{ BIODIGESTORES } & \multirow{2}{*}{ MÉTODO } \\
\cline { 3 - 6 } & & B1 & B2 & B3 & B4 & \\
\hline Metano & $\%$ & 40,29 & 0,24 & 2,94 & 2,45 & \multirow{2}{*}{ cromatografia de gases } \\
\hline
\end{tabular}

Elaborado por: Elaborado por: Malacatus Paúl \& all. Fuente: Elaborado por: Malacatus Paúl \& all.

El biodigestor B1, que contenía únicamente inóculo, produjo biogás con un 40,29\% de metano. En tanto que los biodigestores B2, B3 y B4 produjeron biogás con $0,24 \%, 2,94 \%$ y $2,45 \%$ de metano respectivamente. Con esto se puede interpretar que el principal problema de los biodigestores podría estar relacionado con la composición de los residuos cargados, ya que proceso de DA no llegó a completarse hasta la etapa de metanogénesis.

Es así que se decidió llevar a cabo el Ensayo Tres para confirmar las causas de la baja producción de metano en el Ensayo Dos.

Ensayo Tres: La producción acumulada de biogás obtenido del tratamiento de la FORS del Mercado de Carapungo, fue monitoreado durante 42 días. Los resultados se presentan a continuación:

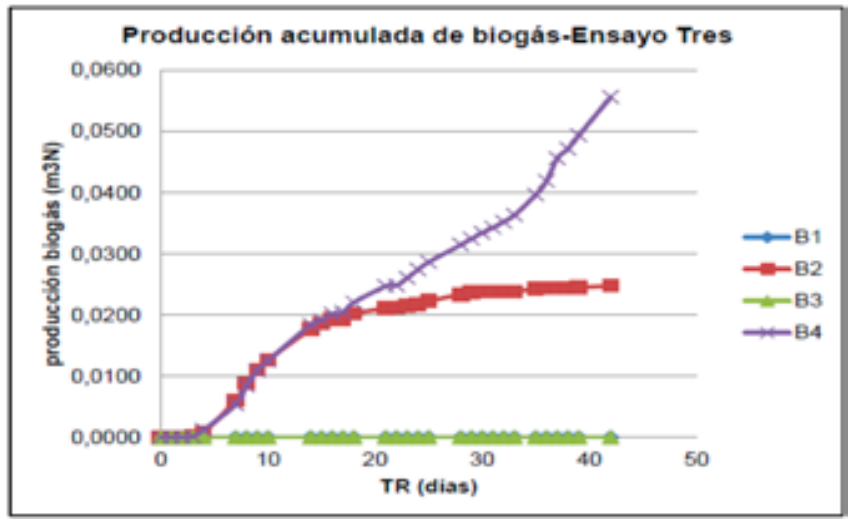

Figura 9 Producción acumulada de biogás-Ensayo Tres 
Al igual que en el Ensayo Dos, los biodigestores B1 y B3 no produjeron biogás. Los biodigestores B2 y B4 tuvieron un comportamiento similar hasta el día 16. Del día 17 al 42 se observa que el biodigestor B4 produce un volumen de biogás mucho mayor en comparación con B2.

A pesar de que se trabajó bajo las mismas condiciones en todos los biodigestores, éstos presentaron comportamientos distintos en cuanto a la producción acumulada de biogás, es así que estos dispositivos experimentales no pueden ser validados para el tratamiento de la FORS.

Además, en este ensayo se monitoreó semanalmente la composición de biogás para cada biodigestor, obteniendo así los datos finales presentados en la siguiente tabla:

Tabla 4 Concentración de metano-Ensayo Tres

\begin{tabular}{|c|c|c|c|c|c|c|}
\hline \multirow{2}{*}{ DIA } & \multirow{2}{*}{ UNIDAD } & \multicolumn{4}{|c|}{ BIODIGESTORES } & \multirow{2}{*}{ MÉTODO } \\
\cline { 3 - 6 } & & B1 & B2 & B3 & B4 & \\
\hline 11 & $\%$ & 12,14 & 12,74 & 13,21 & 8,87 & \\
\hline 21 & $\%$ & 13,98 & 14,92 & 16,33 & 12,45 & $\begin{array}{c}\text { cromatografia } \\
\text { de gases }\end{array}$ \\
\hline 36 & $\%$ & 17,74 & 22,41 & - & 21,92 & \\
\hline
\end{tabular}

Fuente: Elaborado por: Malacatus Paúl \& all.

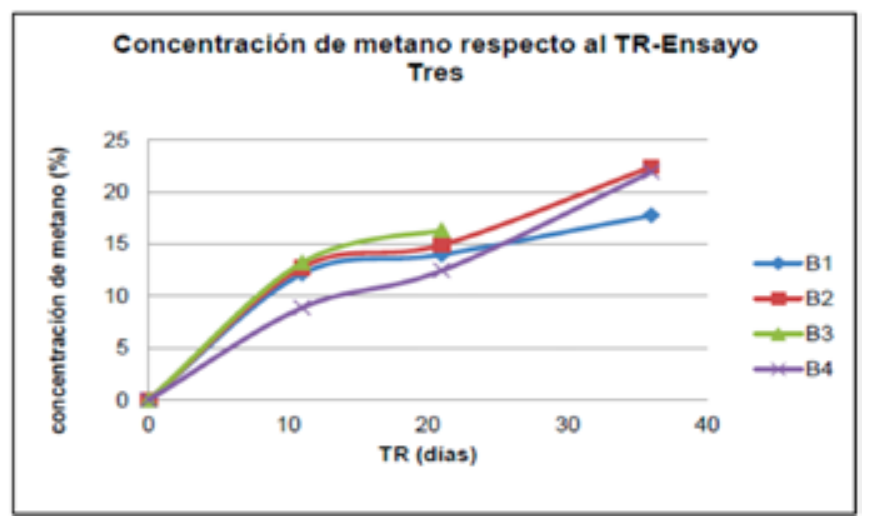

Figura 10 Variación de la concentración de metano respecto al TR-Ensayo Tres

Como se puede observar, la concentración de metano en el biogás aumenta respecto al TR de todos los biodigestores. A pesar de que no se tiene el último dato de concentración de metano en B3, por la falta estándar en el cromatógrafo de gases, se puede deducir que tiene la misma tendencia que los demás biodigestores.

La producción de metano (alrededor del 20\%) es alta tomando en cuenta su bajo tiempo de retención (42 días), lo cual es comparable con la elevada concentración de metano (40,29\%) obtenida en el biodigestor B1 del Ensayo Dos.

Dado que todos los biodigestores en el Ensayo Tres y el biodigestor B1 del Ensayo Dos se cargaron úni- camente con estiércol, se ratifica que la baja producción de metano en los biodigestores cargados con FORS del Ensayo Dos se debe a la composición inhibidora de dicho sustrato por su considerable porcentaje de cítricos, y por otra parte a la relación FORS-inóculo empleada, ya que de acuerdo con FAO (2011, citado en Cevallos \& Hidalgo, 2013), una sobrecarga puede dar como resultado la producción excesiva de ácidos orgánicos, siendo éstos ácidos grasos volátiles u otros compuestos intermedios inhibidores (Pascual et al., 2011), durante la primera fase de la digestión que provoca una disminución en el pH del biorreactor y posterior un fallo en el proceso de la producción del metano (FAO 2011, citado en Cevallos \& Hidalgo, 2013).

Éste fenómeno ya ha sido reportado por Bogotá et al. (2008), quienes establecen en su investigación que la causa para la inhibición del crecimiento de bacterias metanogénicas fue el descenso de $\mathrm{pH}$.

Es así que, la DA dentro de los biodigestores fue incompleta, lo que desencadenó la inhibición de la metanogénesis, llegando únicamente hasta la etapa de acidogénesis. Por lo tanto, la comparación del potencial de biogás entre las técnicas estudiadas no fue posible, dado que el gas obtenido bajo las condiciones anteriormente expuestas, posee una concentración de metano menor al 3\% y para ser considerado biogás ésta debería estar entre 55 y 70\% (FAO, 2011).

\section{Conclusiones}

- No se genera mayor potencial de biogás a partir de la recirculación en dos etapas, dado que no fue posible la comparación entre las técnicas de digestión anaerobia (DA) experimentadas.

- Los residuos generados en el Mercado de Carapungo, en su mayoría son orgánicos $(72,72 \%)$ y contienen un alto porcentaje de sólidos volátiles (76,01\%), por lo que se establece que éstos pueden tener un alto potencial para generar biogás bajo condiciones favorables de relación FORS-inóculo y del tipo de reactor empleado. Sin embargo, esta FORS contiene un $15,58 \%$ de cítricos, lo que puede inhibir el proceso de DA.

- En el diseño de los biodigestores experimentales utilizando bidones de cierre hermético, además de los accesorios implementados, es necesario generar un sello hidráulico al dar la vuelta a los bidones para garantizar la hermeticidad de los mismos. 
- No es posible comparar el potencial de biogás entre las técnicas estudiadas dado que el gas obtenido posee un porcentaje de metano menor al 3\%, el cual no es representativo para ser considerado biogás, debido a que el proceso de DA llegó únicamente hasta la etapa de acidogénesis.

- La técnica de DA batch, independientemente de que tenga o no recirculación, es limitada para el tratamiento de la FORS del Mercado de Carapungo, debido al shock originado por la relación FORS- inóculo en la alimentación de los biodigestores, así como también por la composición de los residuos sólidos orgánicos del mencionado mercado, lo cual conlleva a la inhibición del crecimiento de bacterias metanogénicas.

\section{Referencias bibliográficas}

[1] Bogotá, J., Díaz, S. \& Ramos, P. (2008). Montaje y puesta en marcha de dos biodigestores anaerobios con residuos orgánicos generados en la central de mercado "Plaza Kennedy" en Bogotá. (Tesis de pregrado). Facultad de Ingeniería Ambiental, Universidad Manuela Beltrán, Bogotá, Colombia.

[2] Bouallagui, H., Touhami, Y., Ben Cheikh, R. \& Hamdi, M. (2005). Bioreactor performance in anaerobic digestion of fruit and vegetable wastes. Process Biochemistry, 40, pp. 989-995.

[3] British Standards Institution (2009). Determination of the content of volatile matter (BS EN 15148:2009). London, UK.

[4] Castillo, M. \& Hardter U. (2014). Gestión Integral de Residuos Sólidos en Regiones Insulares. Quitto: WWF. Deublein, D., \& Steinhauser A. (2008). Biogas from waste and renewable resources: An introduction. Weinheim: Wiley-VCH-Verlag.

[5] Diario Oficial de la Federación (1982). Alimentos. Determinación de humedad - Método rápido de la termobalanza (NMX-F-428-1982). México, D.F.

[6] Diario Oficial de la Federación (1985). Norma de Determinación de Peso Volumétrico in situ (NMX-AA-19- 1985). México, D.F.

[7] Diario Oficial de la Federación (1985). Norma de Muestreo -Método de Cuarteo (NMX-AA-15-1985). México, D.F.

[8] Diario Oficial de la Federación (1985). Norma de Selección y Cuantificación de subproductos (NMX-AA-221985). México, D.F.
[9] Díaz de Basurto, A. (2013). Diseño, construcción y puesta en marcha de un biodigestor anaerobio con residuos orgánicos generados en el Mercado de Tiquipaya (Bolivia). (Tesis de maestría). Escuela Técnica Superior de Ingenieros de Caminos, Canales y Puertos de Barcelona, Universidad Politécnica de Cataluña, Barcelona, España.

[10] Forster-Carneiro, T., Pérez, M., Romero, L. \& Sales, D. (2007). Dry-thermophilic anaerobic digestion of organic fraction of the municipal solid waste: Focusing on the inoculum sources. Bioresource Technology, 98, pp. 3195-3203.

[11] Lohri, C. (2009). Research on anaerobic digestion of organic solid waste at household level in Dar es Salaam, Tanzania. (Bachelor Thesis). ZurichUniversity of Applied Sciences in collaboration with EAWAG (Swiss Federal Institute of Aquatic Science and Technology).

[12] Luste, S. \& Luostarinen, S. (2010). Anaerobic codigestion of meat-processing by-products and sewage sludge Effect of hygienization and organic loading rate. Biore-source Technology, 101, pp. 2657-2664.

[13] Martí-Herrero, J., Chipana, M., Cuevas, C., Paco, G., Serrano, V., Zymla, B., Heising, K., Sologuren, J. \& Gamarra, A. (2014). Low cost tubular digesters as appropriate technology for widespread application: results and lessons learned from Bolivia. Renewable Energy, 71, pp. 156-165.

[14] Martí-Herrero, J., Soria, G., Díaz-de-Basurto, A. \& Álvarez, R. (2015). Firsts results from a new low cost digester to treat the organic fraction of municipal solid waste (artículo no publicado). Tiquipaya: para ser publicado en el Anaerobic Digestion World Congress, Chile, noviembre, 2015.

[15] Mata-Alvarez, J. (2003). Biomethanization of the organic fraction of municipal solid wastes. IWA publishing company, London.

[16] Qiao, W., Yan, X., Ye, J., Sun, Y., Wang, W., Zhang, Z. (2011). Evaluation of biogas production from different biomass wastes with/without hydrothermal pretreatment. Renewable Energy, 36, 3313-3318.

[17] Rittmann, B. \& McCarty, P. (2001). Environmental Biotechnology: Principles and Applications. McGrawHill Series in Water Resources and Environmental Engineering 